\title{
Adenoma of the nipple: A clinicopathological report of 13 cases
}

\author{
MAURIZIO DI BONITO ${ }^{1}$, MONICA CANTILE ${ }^{1}$, FRANCESCA COLLINA ${ }^{1}$, MASSIMILIANO D'AIUTO $^{2}$, \\ GIUSEPPINA LIGUORI ${ }^{1}$, ROSSELLA DE CECIO ${ }^{1}$ and GERARDO BOTTI ${ }^{1}$
}

Departments of ${ }^{1}$ Pathology and ${ }^{2}$ Senology, National Cancer Institute, Fondazione Pascale Hospital, Naples I-80131, Italy

Received May 30, 2013; Accepted December 6, 2013

DOI: $10.3892 / \mathrm{ol} .2014 .2000$

\begin{abstract}
Adenoma of the nipple (AN) represents a rare benign mammary proliferation of lactiferous ducts. It appears as an erosive or ulcerative lesion, which in a number of cases is associated with a serous/hematic secretion. AN may be clinically confused with Paget's disease and histologically with invasive breast carcinoma or breast cancer precursor lesions. Therefore, the histological and immunophenotypic analysis is essential for the differential diagnosis. The present study describes the histopathological characteristics of a first case series of AN.
\end{abstract}

\section{Introduction}

Adenoma of the nipple (AN) is a rare benign epithelial tumor of the nipple ducts. It generally occurs unilaterally and arises at an average age of between 43-45 years, predominantly in females and rarely in males and adolescents (1-3). The lesion, also known as erosive adenoma and florid papillomatosis, appears similar to a hard-elastic nodule that deforms the nipple, causing swelling or erosion with serous or hematic secretion. AN is often confused with Paget's disease (4) and the differential diagnosis with breast carcinoma is often difficult $(5,6)$.

AN appears histologically as an extremely heterogeneous tumor entity, particularly due to the following various patterns of growth associated with it: i) 'sclerosing papillomatosis pattern', often indistinguishable from sclerosing papilloma; ii) 'papillomatosis pattern', florid papillary hyperplasia of ductal epithelium; iii) 'adenosis pattern', evident myoepithelial hyperplasia; and iv) 'mixed proliferative pattern', combination of three patterns (metaplasia of ducts with cysts, apocrine metaplasia and acanthosis of the epithelium) (7).

A larger series previously reported in the literature referred to a group of 42 American patients and a casuistry of 18 Chinese patients $(8,9)$, collected over several decades,

Correspondence to: Dr Monica Cantile, Department of Pathology, National Cancer Institute, Fondazione Pascale Hospital, Via Mariano Semmola, Naples I-80131, Italy

E-mail: monicantile@libero.it

Key words: nipple adenoma, myoepithelial cells, immunophenotype which did not include Italian patients. Only sporadic case reports have been previously described $(2,10)$.

The present study described a series of 13 cases of AN with clinicopathological features, collected within a decade, highlighting the incidence of this benign lesion in the population of Southern Italy. In addition, the requirement of a careful morphological analysis, associated with a relevant immunophenotypic panel, for the recognition of this lesion and differential diagnosis with other breast malignant neoplasms was highlighted.

\section{Materials and methods}

Clinical information. Cases were selected from the pathological files of the National Cancer Institute, Fondazione Pascale Hospital (Naples, Italy) between January 2003 and April 2013. The World Health Organization (WHO) criteria was strictly applied to establish the diagnosis of AN. Clinical information was recovered from clinical files and a total of 13 cases were identified. All patients signed an informed consent form according to the institutional regulations.

Immunophenotype analysis. The formalin-fixed, paraffin-embedded (FFPE) tissue block specimens were sectioned (3- $\mu \mathrm{m}$ thick), deparaffinized and rehydrated. Each section was stained with hematoxylin and eosin and then used for immunostaining. Immunohistochemical analyses were performed using an autostainer (BenchMark XT system; Ventana Medical Systems, Inc., Tucson, AZ, USA) according to the manufacturer's instructions. The following anti-human primary antibodies were used: p63 (Santa Cruz Biotechnology, Inc,. Santa Cruz, CA, USA), caldesmon, calponin, $\alpha$-smooth muscle actin, CD10, cytokeratin (CK) 5/6 (DakoCytomation, Glostrup, Denmark) and CK8/18 (Novocastra, Newcastle, UK) (Table I).

Stained sections were evaluated by two different pathologists using uniform criteria. Discrepancies were resolved through simultaneous evaluation and discussion of the results. Single-marker expression was recorded as negative/positive and high/low level, following consideration of the expression in reactive surrounding tissue compared with tumoral cells and the specific cut-off of each marker.

\section{Results}

Clinicopathological features. All AN patients were admitted to the National Cancer Institute, Fondazione Pascale Hospital 
Table I. Antibody panel for immunohistochemistry analysis.

\begin{tabular}{llll}
\hline Antibody & \multicolumn{1}{c}{ Source } & Clone & Dilution \\
\hline p63 & Rabbit polyclonal & Sc-8343 & $1: 200$ \\
h-CALD1 & Mouse monoclonal & h-cd & $1: 400$ \\
Calponin & Mouse monoclonal & CALP & $1: 600$ \\
$\alpha$-smooth muscle actin & Mouse monoclonal & 1 A & Prediluted \\
CD10 & Mouse monoclonal & $56 C 6$ & $1: 50$ \\
CK5/6 & Mouse monoclonal & D5/16B4 & Prediluted \\
CK8/18 & Mouse monoclonal & 5D3-R-7-CE & Prediluted
\end{tabular}

h-CALD1, h-caldesmon 1; CK, cytokeratin.

Table II. Clinicopathological features of patients.

\begin{tabular}{|c|c|c|c|c|c|c|c|c|c|c|}
\hline \multirow[b]{2}{*}{ Patient } & \multirow{2}{*}{$\begin{array}{l}\text { Age, } \\
\text { years }\end{array}$} & \multirow{2}{*}{$\begin{array}{l}\text { Tumor } \\
\text { size, cm }\end{array}$} & \multirow[b]{2}{*}{ Growth pattern } & \multicolumn{6}{|c|}{ Myoepithelial markers } & \multirow[b]{2}{*}{ CK8/18 } \\
\hline & & & & p63 & CALD1 & CALP1 & M-actin & CD10 & CK5/6 & \\
\hline 1 & 38 & $0.9 \times 1.2$ & Papillomatosis pattern & + & $+/-$ & + & + & + & + & + \\
\hline 2 & 20 & $0.8 \times 1.1$ & Papillomatosis pattern & + & + & + & + & + & + & + \\
\hline 3 & 40 & $1.6 \times 1.3$ & Papillomatosis pattern & + & $+/-$ & + & + & + & + & + \\
\hline 4 & 31 & $0.7 \times 1.5$ & papillomatosis pattern & + & $+/-$ & - & + & + & + & + \\
\hline 5 & 51 & $1.2 \times 1.3$ & Mixed prolif. pattern & + & $+/-$ & - & + & + & + & + \\
\hline 6 & 42 & $0.7 \times 1.1$ & Papillomatosis pattern & + & - & + & + & + & + & + \\
\hline 7 & 37 & $1.3 \times 0.9$ & Papillomatosis pattern & + & $+/-$ & + & + & + & + & + \\
\hline 8 & 37 & $0.5 \times 1.2$ & Papillomatosis pattern & + & $+/-$ & + & + & + & + & + \\
\hline 9 & 31 & $1.3 \times 1.5$ & Papillomatosis pattern & + & + & $+/-$ & + & + & + & + \\
\hline 10 & 44 & $0.8 \times 1.2$ & Papillomatosis pattern & + & $+/-$ & + & + & + & + & + \\
\hline 11 & 44 & $1.1 \times 1.3$ & Papillomatosis pattern & + & + & $+/-$ & + & + & + & + \\
\hline 12 & 42 & $0.8 \times 1.3$ & Mixed prolif. pattern & + & + & $+/-$ & + & + & + & + \\
\hline 13 & 42 & $1.2 \times 1.2$ & Adenosis pattern & + & + & - & + & + & + & + \\
\hline
\end{tabular}

${ }^{a}$ Epithelial marker. CALD1, caldesmon 1; CALP1, calponin 1; M-actin, $\alpha$-smooth muscle actin; CK, cytokeratin; prolif., proliferation.

following a physical examination revealing a well-defined erosive tumor, often serousanguineous, of the breast nipple. Mammography and ultrasonography revealed no mass lesions and calcifications in the two breasts. In total, three cases appeared clinically as Paget's disease. A total excision of the nipple and areola with an underlying portion of breast tissue was obtained. All clinicopathological parameters of patients are included in Table II.

In summary, all patients were female, with an age range of 20-51 years and an average age of 38 years. The medium size of the lesions was between 0.8 and $1.5 \mathrm{~cm}$.

Histopathological observations. Macroscopically, all lesions presented in the retroareolar region, with no encapsulated nodules and infiltrative margins (Fig. 1). The presence of adenomatous proliferation in the stroma of medium and small caliber ducts, coated by a double layer of cells (epithelial and myoepithelial) was detected in all samples. Only one of the 13 cases appeared with ductal carcinoma in situ (DCIS) following the intraoperative examination. The histological features of the 13 lesions were extremely variegated even when the prevalent growth pattern was the papillomatosis pattern with a florid papillary hyperplasia of ductal epithelium. In the majority of cases, the following features were observed: i) presence of fibrosis with distortion of the ducts that may simulate images of pseudo invasion; ii) epithelial hyperplasia with a partial or total obliteration of the lumen; iii) epithelial hyperplasia with intraductal papillary projections; iv) presence of intraductal necrosis; v) presence of cellular monomorphism and/or polymorphism; vi) cellular atypia; and vii) mitosis in $50 \%$ of cases. One case showed an adenosis pattern with myoepitelial hyperplasia and two cases showed a mixed proliferative pattern (Fig. 2).

Immunohistochemical observations. Immunohistochemical studies were performed on all AN specimens. For epithelial cells of the inner layer of ducts, CK8/18 antibodies were used, while myoepithelial cells of the outer layer were highlighted using antibodies against p63, caldesmon, calponin, $\alpha$-smooth muscle actin, CK5/6 and CD10. 


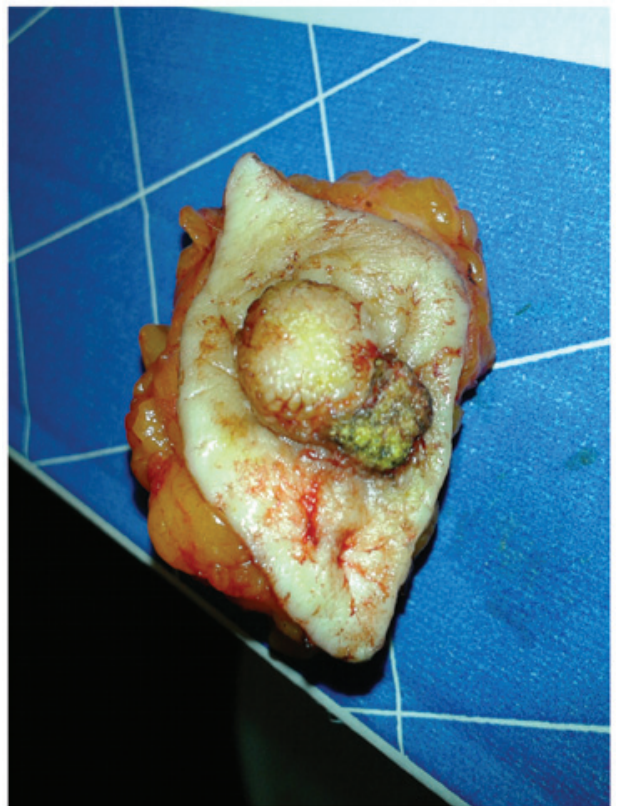

Figure 1. Complete resection of the nipple with erythema, superficial ulceration and crusting.

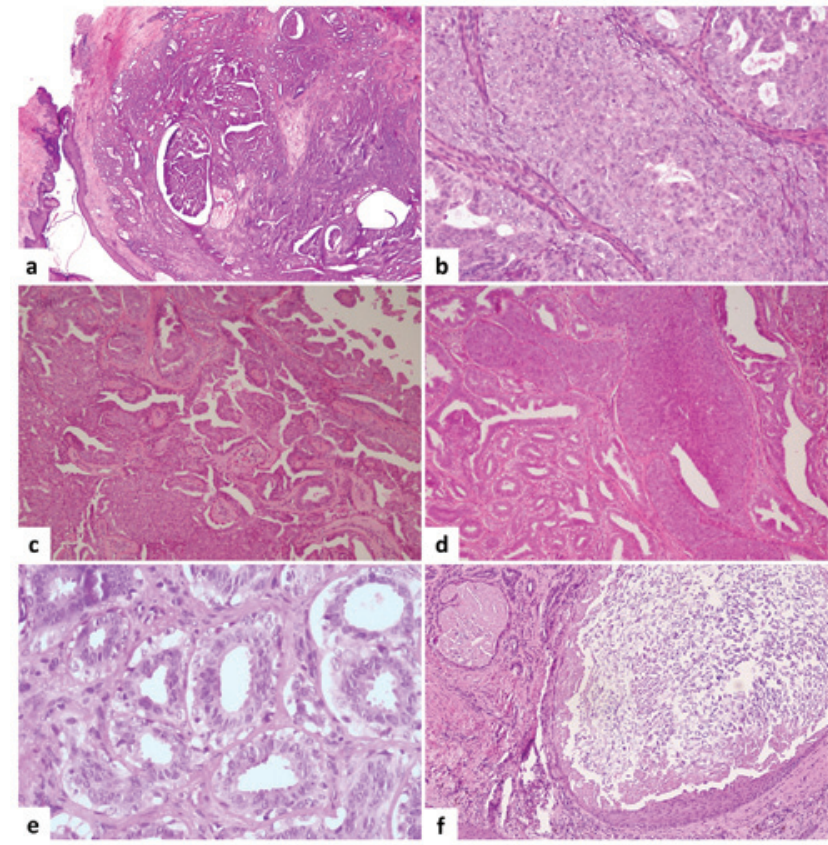

Figure 2. Hematoxylin and eosin morphology. (a) AN lesion overview (magnification, x10). (b) Papillomatosis pattern with usual ductal hyperplasia (magnification, x40). (c) Papillomatosis pattern (magnification, $\mathrm{x} 20$ ). (d) Mixed pattern (papillomatosis and adenosis; magnification, x20). Adenosis pattern with (e) myoepithelial hyperplasia (magnification, $\mathrm{x} 40$ ) and (f) keratocysts (magnification, $\mathrm{x} 20$ ). AN, adenoma of the nipple..

The details of positivity/negativity for several markers is included in Table II and shown in Fig. 3.

\section{Discussion}

$\mathrm{AN}$ is a rare benign tumor of the breast, which originates from the nipple areola complex generally between the fourth or fifth decade of life. This lesion is almost always unilateral

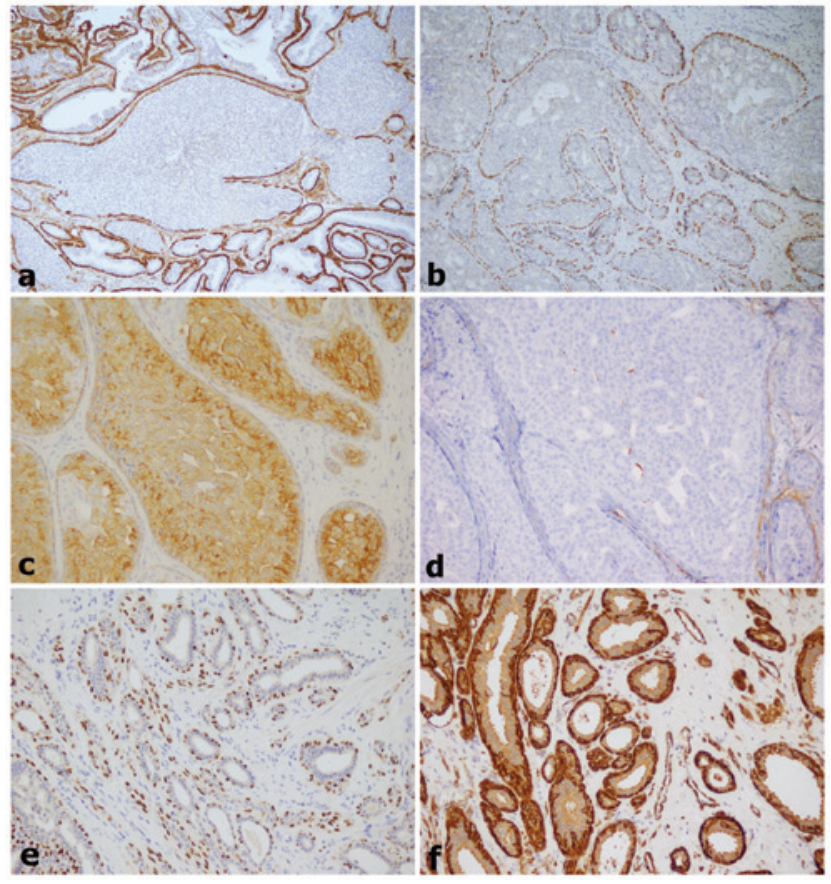

Figure 3. Immunophenotyping. (a) M-actin, (b) p63, (c) cytokeratin 5/6 and (d) cladesmon 1 staining in AN with papillomatosis pattern and (e) p63 and (f) M-actin staining in AN with adenosis pattern (magnification, x20). $\mathrm{M}$-actin, $\alpha$-smooth muscle actin; AN, adenoma of the nipple.

and is often accompanied by a serous/hematic secretion in the nipple. In the WHO classification, AN is defined as 'a compact proliferation of small tubules lined by epithelial and myoepithelial cells, with or without proliferation of the epithelial component, around the collecting ducts of the nipple' (11).

However, there is considerable confusion concerning the terms used to define this lesion, due to the diversity of histological pattern with which it occurs. It has been defined as erosive adenomatosis of the nipple, papillary AN, florid adenomatosis, florid papillomatosis of the nipple, subareolar duct papillomatosis and superficial papillary adenomatosis of the nipple $(2,8,12)$. Since the main feature common to these lesions is adenomatous proliferation in the stroma (small and medium caliber duct proliferation) $(1,4)$, the definition of $\mathrm{AN}$ was preferred in the current study.

Although AN are rare and benign entities, the main issue with these lesions is the differential diagnosis with nipple Paget's disease (clinical and histological diagnosis), DCIS of low-grade, tubular carcinoma, infiltrating syringomatous adenoma and solitary central papilloma subareolar (histological diagnosis) (7).

These lesions are characterized by the presence of two cell populations, an internal layer of cuboidal epithelial cells with an apocrine secretion and an external layer of myoepithelial cells. The presence of a myoepithelial cell layer in neoplastic ducts is considered to be the most important histological observation for distinguishing adenoma from carcinoma. For this reason, the correct immunophenotypic definition, through the use of a panel of specific antibodies for the myoepithelial cells, is always required for the differential diagnosis. Among the frequently used myoepithelial markers are p63, h-caldesmon, 
calponin $1, \alpha$-smooth muscle actin, CK5/6 and CD10 $(13,14)$. The positivity of at least two markers is sufficient for diagnosis. The use of p63 has been largely discussed since it may be extremely useful, particularly for the differential diagnosis with DCIS. In this lesion, the expression of p63 is lost or may appear discontinuous (15). The CK5/6, in addition to myoepithelial cells, is also present within the intraductal epithelial proliferation lesion. In the case of differential diagnosis with atypical ductal hyperplasia and DCIS, positivity for CK5/6 within the ducts is lost (14).

Cytological examination may be performed for diagnosis, but the complete excision of the lesion and examination of FFPE serial sections remains the gold standard for diagnosis. Although the lesion is almost always unilateral, bilateral cases $(16,17)$ and association of $\mathrm{AN}$ with malignant breast carcinoma (18-21) have been previously described. With regard to the probability of a tumor developing from these lesions, no reliable data has been identified in the previous literature $(22,23)$.

To date, few case studies have analyzed the numerous individual case reports for AN. A previous case series of 15 cases was described in 1985 by Brownstein et al (12). Subsequently, the largest case series was presented in 1986 by Rosen and Caicco with 42 selected cases of AN (8). Finally, a case series of $18 \mathrm{AN}$ cases in the Chinese population was described (9). No previous studies have analyzed the incidence of this lesion in Italy. Since 2002, only single case reports have been presented $(2,10)$.

In the present study, a case series of 13 patients was selected from the National Cancer Institute of Fondazione Pascale Hospital database. This was collected within ten years and represented a female population from the Campania region of Southern Italy. The range of ages of the patients recruited in the study corresponds with that described in the previous literature and the mean age was $\sim 38$ years. In addition, the growth pattern frequently found was the papillomatosis pattern with a florid papillary hyperplasia of ductal epithelium. All lesions presented were unilateral and not associated with other malignant diseases of the breast. In all analyzed cases, the definition of the immunophenotypic profile was essential for the correct diagnosis.

In conclusion, although AN may be diagnosed preoperatively by cytological examination and core biopsy, complete excision of the lesion and an adequate histological and immunophenotypic analysis is recommended. This is necessary to discriminate the pseudo invasive pattern that often characterizes this lesion from breast cancer precursors and aggressive carcinoma.

\section{References}

1. Fernandez-Flores $A$ and Suarez-Peñaranda JM: Immunophenotype of nipple adenoma in a male patient. Appl Immunohistochem Mol Morphol 19: 190-194, 2011.

2. Tuveri M, Calò PG, Mocci C and Nicolosi A: Florid papillomatosis of the male nipple. Am J Surg 200: e39-e40, 2010.

3. Ishii N, Kusuhara M, Yasumoto S and Hashimoto T: Adenoma of the nipple in a Japanese man. Clin Exp Dermatol 32: 448-449, 2007.

4. Healy CE, Dijkstra B, Walsh M, Hill AD and Murphy J: Nipple adenoma: a differential diagnosis for Paget's disease. Breast J 9: 325-326, 2003.

5. Aftab K and Idrees R: Nipple adenoma of breast: a masquerader of malignancy. J Coll Physicians Surg Pak 20: 472-474, 2010.

6. Da Costa D, Taddese A, Cure ML, Gerson D, Poppiti R Jr and Esserman LE: Common and unusual diseases of the nipple-areolar complex. Radiographics 27 (Suppl 1): S65-S77, 2007.

7. Rosen PP: Rosen's breast pathology. 3rd Edition Lippincott Williams \& Wilkins (Wolters Kluwer), Philadelphia, 2009.

8. Rosen PP and Caicco JA: Florid papillomatosis of the nipple. A study of 51 patients, including nine with mammary carcinoma. Am J Surg Pathol 10: 87-101, 1986.

9. Yang GZ, Li J and Ding HY: Nipple adenoma: report of 18 cases with review of literatures. Zhonghua Bing Li Xue Za Zhi 38: 614-616, 2009 (In Chinese).

10. Interlandi A and Busacca G: Adenomas of the nipple. Minerva Chir 57: 699-702, 2002 (In Italian).

11. Tavassoli FA and Devilee P (eds): World Health Organization Classification of Tumors. Pathology and Genetics of Tumours of the Breast and Female Genital Organs. IARC Press, Lyon, 2003.

12. Brownstein MH, Phelps RG and Magnin PH: Papillary adenoma of the nipple: analysis of fifteen new cases. J Am Acad Dermatol 12: 707-715, 1985.

13. Batistatou A, Stefanou D, Arkoumani E and Agnantis NJ: The usefulness of p63 as a marker of breast myoepithelial cells. In Vivo 17: 573-576, 2003.

14. Dewar R, Fadare O, Gilmore H and Gown AM: Best practices in diagnostic immunohistochemistry: myoepithelial markers in breast pathology. Arch Pathol Lab Med 135: 422-429, 2011.

15. Werling RW, Hwang $\mathrm{H}$, Yaziji $\mathrm{H}$ and Gown AM: Immunohistochemical distinction of invasive from non-invasive breast lesions: a comparative study of p63 versus calponin and smooth muscle myosin heavy chain. Am J Surg Pathol 27: 82-90, 2003.

16. Bergdahl L, Bergman F, Rais O and Westling P: Bilateral adenoma of nipple. Report of a case. Acta Chir Scand 137: 583-586, 1971.

17. Citoler P, Broer KH and Zippel HH: Bilateral adenoma of nipple. Geburtshilfe Frauenheilkd 33: 729-731, 1973 (In German).

18. Rao P and Shousha S: Male nipple adenoma with DCIS followed 9 years later by invasive carcinoma. Breast J 16: 317-318, 2010.

19. Jones MW and Tavassoli FA: Coexistence of nipple duct adenoma and breast carcinoma: a clinicopathologic study of five cases and review of the literature. Mod Pathol 8: 633-636, 1995.

20. Hansen U and Rank F: Adenoma of the nipple and concomitant breast cancer. Ugeskr Laeger 147: 1852-1853, 1985 (In Danish).

21. Bhagavan BS, Patchefsky A and Koss LG: Florid subareolar duct papillomatosis (nipple adenoma) and mammary carcinoma: report of three cases. Hum Pathol 4: 289-295, 1973.

22. Ermilova VD and Seredin VP: Adenoma of the nipple with malignant degeneration. Arkh Patol 49: 59-61, 1987 (In Russian).

23. Gudjónsdóttir A, Hägerstrand I and Ostberg G: Adenoma of the nipple with carcinomatous development. Acta Pathol Microbiol Scand A 79: 676-680, 1971. 